Published in final edited form as:

Curr Opin Gastroenterol. 2018 September ; 34(5): 317-321. doi:10.1097/MOG.0000000000000460.

\title{
Surgical treatment of pain in chronic pancreatitis
}

\author{
Mariya E. Skube and Greg J. Beilman \\ Department of Surgery, University of Minnesota, Minneapolis, Minnesota, USA
}

\begin{abstract}
Purpose of review-Patients suffering from chronic pancreatitis often require surgical intervention to treat their disease. This review discusses surgical options as well as reviews current trends and research in the operative management of chronic pancreatitis.

Recent findings-Relevant current topics in the field include the appropriate timing of surgery as well as the relative benefits of various procedures, particularly duodenum-preserving pancreatic head resection versus pancreaticoduodenectomy. Multiple studies have found that surgery earlier in the disease course results in improved outcomes. Furthermore, the recent literature reports similar outcomes of duodenum-preserving pancreatic head resection when compared with pancreaticoduodenectomy.
\end{abstract}

Summary-It is important for treating clinicians to be well versed on the interplay of medical, endoscopic, and surgical strategies to carefully tailor a patient's treatment plan. Each patient warrants careful consideration and an individualized approach in collaboration with multidisciplinary colleagues.

\section{Keywords}

chronic pancreatitis; pancreatectomy; surgical decision-making

\section{INTRODUCTION}

For $40-75 \%$ of chronic pancreatitis patients, the pain and related sequalae of their disease ultimately lands them in the operating room [1,2]. The timing of operative intervention and the surgical procedure of choice are carefully determined by individual patient factors such as underlying cause, duration of disease, and ductal morphology. This article will provide a brief overview of the surgical options for management of pain in chronic pancreatitis as well as discuss the current salient topics in the field.

\section{SURGICAL MANAGEMENT OPTIONS}

Traditionally, the arsenal of surgical techniques for treating chronic pancreatitis has included options for both drainage and resection $[1,3,4 \boldsymbol{-}]$. A description of the most relevant

\footnotetext{
Correspondence to Greg J. Beilman, MD, Department of Surgery, University of Minnesota, 420 Delaware St SE, MMC 195, Minneapolis, MN 55445, USA. Tel: +1 612626 6122; beilman@umn.edu.

Conflicts of interest

There are no conflicts of interest.
} 
procedures is provided in Table 1. Although each patient case merits an individualized approach, there are some basic guidelines to the appropriate surgical approach for chronic pancreatitis patients. Figure 1 provides a simple guide to surgical decision-making for these patients [5].

\section{MANAGEMENT TRENDS AND NEW LITERATURE}

In the past few years, the work in the field has a number of recurring themes. Reflecting the trends of recent publications and debates within the field, the emphasis of the following discussion will be the timing of surgery with particular consideration of the interplay of endoscopic and surgical approaches, the evaluation of duodenum-preserving pancreatic head resection (DPPHR), and the splanchotomy approach to pain management.

\section{TIMING OF SURGERY IN A MINIMALLY INVASIVE ERA}

A crucial aspect of surgical success in the treatment of chronic pancreatitis is the careful selection of the appropriate timing of surgical intervention. In 2012, the Dutch Pancreatitis Study Group reported on pain relief after surgery for chronic pancreatitis [6]. They found that 3 years or less of pain $(P=0.03)$, absence of preoperative narcotic use $(P=0.006)$, and five or less endoscopic procedures $(P=0.05)$ were independent predictors of postoperative pain relief. Of note, total pancreatectomy patients were not included. These findings challenged the theory of exhausting medical management prior to operative intervention and suggested early surgical exposure may be advantageous. Following this report, Yang et al. published a single institution's experience with the surgical treatment of pain associated with chronic pancreatitis to assess optimal timing of surgery [7]. Their research revealed that duration of chronic pancreatitis prior to operating of 26.5 months was the cutoff threshold (area under the curve 0.66) for their series of 66 patients in having successful pain relief.

Advances in the field of endoscopic management present further challenges in determining the best timing of surgery. Often, endoscopy can relieve pain and associated symptoms for many patients and provide a less invasive treatment option. These capabilities also present an additional challenge in determining the ideal timing to proceed with a surgical approach. As the aforementioned studies reveal, earlier surgical intervention may actually improve rates of postoperative pain relief. Furthermore, multiple studies have revealed that increasing numbers of endoscopic intervention and/or stent placement predict less pain relief postoperatively $[6,8]$. For example, in a review of 581 total pancreatectomy patients by Chinnakotla et al., multivariate analysis indicated that a previous stent placement $[P=0.055$, odds ratio (OR) 1.93], greater than two stents ( $P=0.020$, OR 2.73), and prior sphincterotomy $(P=0.020$, OR 1.76$)$ were associated with narcotic use at 1 year after surgical intervention [8].

Nonetheless, endoscopy plays an important role in the management of chronic pancreatitis. For many patients, endoscopy provides significant pain relief and promising disease management. Jafri et al. compiled a meta-analysis of 16 studies investigating the efficacy of endotherapy in relieving chronic pancreatitis pain [ ${ }^{\mathbf{m}}$ ]. Although immediate pain relief was reported as high [88\%, 95\% confidence interval (CI) $81-94 \%]$, this down trended to 
$67 \%$ (95\% CI 58-76\%) at long-term follow-up (mean of 47 months). The complication rate was $8 \%$. Patients with intraductal stones in the pancreatic ahead as well as main duct strictures are frequently ideal endoscopic candidates [10]. Endoscopic maneuvers are also often employed in patients with features consistent with both recurrent acute pancreatitis and chronic pancreatitis [11].

Although the literature is admittedly sparse, when compared head to head, surgery currently has the long-term advantage over endoscopy. A Cochrane review of three randomized controlled trials comparing endoscopic and surgical management found that surgical patients had greater and more durable pain relief. At long-term follow-up ( 25 years), the risk ratio of pain relief was 1.56 (95\% CI 1.18-2.05) in the surgical patients with comparable rates of morbidity and mortality [12]. The true challenge is early recognition of patients with disease characteristics not amenable to endoscopic therapy alone, and rigorous clinical trials to provide clearer guidance are needed [13 ${ }^{\mathbf{m}}$. In order to reconcile the advantages of endoscopic therapy with the benefit of early surgery, at the authors' institution a multidisciplinary committee comprised clinicians from surgery, gastroenterology, endocrinology, psychology, and pain backgrounds discuss cases weekly to determine the ideal approach.

\section{DUODENUM-PRESERVING PANCREATIC HEAD RESECTION}

Not only does determining surgical timing pose challenges for the clinician but also selecting the appropriate surgical procedure can present an additional hurdle in the management of chronic pancreatitis patients [14 ]. One area of particular interest has been weighing the relative benefits of partial pancreaticoduodenectomy compared with DPPHR. To this end, a number of recent publications have investigated the role of DPPHR [3,15 , 16,17]. The European ChroPac randomized controlled trial compared quality of life at 2 years postoperatively for patients randomized to pancreaticoduodenectomy and DPPHR groups at 18 hospitals [15 ${ }^{m}$. One hundred and 25 patients were randomized to each group with slightly lower numbers included in final analysis (pancreaticoduodenectomy $n=111$, DPPHR $n=115)$. There was not a difference in reported quality of life $(P=0.284)$ at the designated time point. Secondarily, rates of adverse events were similar between the two groups. The DPPHR group however did have an increased rate of readmissions during the follow-up period (27 versus $11 \%, P=0.002$ ).

Apart from this report on the ChroPac trial, there was also a recent Cochrane reviewing assessing pancreaticoduodenectomy versus DPPHR [16]. Analysis of five eligible trials revealed no difference in mortality, adverse events, or quality of life; however, all the trials were single institution studies and were noted to have low or very low quality of evidence. The length of hospital stay however was lower in the DPPHR patients by $1-5$ days. Further delineating the distinct duodenum-preserving pancreatic head resection strategies, Zhao et al. conducted a systematic review and meta-analysis of the DPPHR versus pancreaticoduodenectomy as well as Beger versus pancreaticoduodenectomy, Frey versus pancreaticoduodenectomy, and Beger versus Frey [18]. DPPHR was found to have a lower operative time $(P<0.00001)$, less blood transfusions $(P=0.02)$, and a shorter length of stay $(P=0.0002)$ compared with pancreaticoduodenectomy. They also found short-term 
morbidity to be lower in the DPPHR group $(P=0.0007)$. Pain relief and quality-of-life outcomes were similar among the groups. Differences in exocrine insufficiency existed more frequently in the pancreaticoduodenectomy group in the studies with shorter follow-up $<<60$ months); however, this did not persist at greater than 60 months of follow-up. Endocrine outcomes were similar.

Investigating variations within the DPPHR procedure itself, Klaiber et al. reported a 10-year followup of a randomized controlled trial comparing the original DPPHR (Beger procedure) to the Berne modification [3]. Sixty-two percent $(n=40)$ of the original cohort had followup data available. There were no long-term differences in the quality-of-life parameters, and both groups reported improved quality of life. Functional outcomes, including daily pain and reoperation among other parameters, were also comparable. As the original Beger procedure is more technically challenging, these results suggest that the Berne modification presents an equally effective alternative.

In summary, the recent literature still indicates mixed results in regards to the optimal surgical intervention, and additional surgical trials are warranted [14 ${ }^{-}$. The recent studies do suggest that for surgical candidates with disease of the pancreatic head, procedures such as DPPHR and Frey result in similar outcomes to pancreaticoduodenectomy and thus may be preferable in order to salvage the duodenum and its key structural and enteroendocrine functions $\left[3,1{ }^{\square}, 16,19,20\right]$. Furthermore, there is a large body of evidence to support the role of total pancreatectomy with islet autotransplantation (TPIAT) in chronic pancreatitis patients with small duct or minimal change disease and intractable pain, and the experience is also growing in patients with recurrent acute and hereditary pancreatitis, including in the pediatric population $[8,21-25,26 \boldsymbol{\square}, 27 \boldsymbol{\square}$. As surgeons who treat chronic pancreatitis are well aware, each patient warrants careful consideration and an individualized approach in collaboration with multidisciplinary colleagues.

\section{SPLANCHNOTOMY}

Seeking surgical relief from pancreatic pain has not been isolated to intra-abdominal procedures. A few recent reports detail experience with splanchnotomy, or splanchnicectomy, from a thoracic approach $[28,29,30]$. Although individual techniques vary, this procedure entails bilateral transection of the greater thoracic splanchnic nerve that contains both sympathetic and visceral afferent fibers. A retrospective review of 123 patients within a single system found that although narcotic pain medication use and hospitalizations decreased in both pancreatic cancer and chronic pancreatitis patients after thoracoscopic splanchnotomy; the effect was more profound in cancer patients [28 $]$. Two other small case series reported successful pancreatic pain relief and minimal complications using this minimally invasive approach to pain control $[29,30]$. The durability of this procedure and comparison with alternate modalities is yet to be clearly elucidated. What this procedure does represent however is that a definite solution for treating the pain of chronic pancreatitis remains elusive, and the field continues to evolve to produce optimal outcomes for patients. 


\section{CONCLUSION}

Chronic pancreatitis is a challenging disease that produces great suffering for the patient and perplexing management decisions for the clinician. It is important for treating clinicians to be well versed on the interplay of medical, endoscopic, and surgical strategies to carefully tailor a patient's treatment plan. Although the timing and technique of surgical intervention must be carefully weighed, there is mounting evidence that pursuing surgery in the first few years of disease for the appropriate candidates provides durable pain relief and improved quality of life in chronic pancreatitis patients.

\section{Acknowledgments}

Financial support and sponsorship

M.E.S. is supported by grant numbers NIH/NIDDK T32DK108733.

\section{REFERENCES AND RECOMMENDED READING}

Papers of particular interest, published within the annual period of review, have been highlighted as:

of special interest

- of outstanding interest

1. Paniccia A, Edil BH. The management of chronic pancreatitis In: Cameron JL, Cameron AM, editors. Current surgical therapy, 12th ed. Philadelphia, PA: Elsevier; 2017 pp. 532-538.

2. Tillou JD, Tatum JA, Jolissaint JS, et al. Operative management of chronic pancreatitis: a review. Am J Surg 2017; 214:347-357. [PubMed: 28325588]

3. Klaiber U, Alldinger I, Probst P, et al. Duodenum-preserving pancreatic head resection: 10-year follow-up of a randomized controlled trial comparing the Beger procedure with the Berne modification. Surgery 2016; 160:127-135. [PubMed: 27106794]

4. Tan CL, Zhang H, Yang M, et al. Role of original and modified Frey's procedures in chronic pancreatitis. World J Gastroenterol 2016; 22:10415-10423. [PubMed: 28058022] This study reports on similar outcomes from the original Frey versus the modified Frey (more limited posterior resection) at a single institution.

5. Beilman G Pancreatitis: is TPIAT the answer for treatment of chronic pancreatitis? Nat Rev Gastroenterol Hepatol 2014; 11:144-145. [PubMed: 24492275]

6. Ahmed Ali U, Nieuwenhuijs VB, van Eijck CH, et al. Clinical outcome in relation to timing of surgery in chronic pancreatitis: a nomogram to predict pain relief. Arch Surg 2012; 147:925-932. [PubMed: 23117832]

7. Yang CJ, Bliss LA, Freedman SD, et al. Surgery for chronic pancreatitis: the role of early surgery in pain management. Pancreas 2015; 44:819-823. [PubMed: 25882695]

8. Chinnakotla S, Beilman GJ, Dunn TB, et al. Factors predicting outcomes after a total pancreatectomy and islet autotransplantation lessons learned from over 500 cases. Ann Surg 2015; 262:610-622. [PubMed: 26366540]

口1 9. Jafri M, Sachdev A, Sadiq J, et al. Efficacy of endotherapy in the treatment of pain associated with chronic pancreatitis: A systematic review and metaanalysis. JOP 2017; 18:125-132. [PubMed: 28966569] This is a systematic review evaluating the efficacy of endotherapy in the treatment of pain associated with chronic pancreatitis, finding that there is a benefit to endotherapy however with decreasing efficacy over time.

10. Majumder S, Chari ST. Chronic pancreatitis. Lancet 2016; 387:1957-1966. [PubMed: 26948434] 
11. Roberts JR, Romagnuolo J. Endoscopic therapy for acute recurrent pancreatitis. Gastrointest Endosc Clin N Am 2013; 23:803-819. [PubMed: 24079791]

12. Ahmed Ali U, Pahlplatz JM, Nealon WH, et al. Endoscopic or surgical intervention for painful obstructive chronic pancreatitis. Cochrane Database Syst Rev 2015; (3):CD007884.

13. Windsor JA, Reddy ND. Endoscopic vs. surgical interventions for painful chronic pancreatitis: What is needed for future clinical trials. Clin Transl Gastroenterol 2017; 8:e213. [PubMed: 28079861] This study discusses the most recent randomized controlled trials evaluating endoscopic versus surgical management of chronic pancreatitis.

14. Bellin MD, Beilman GJ. Surgical trials for chronic pancreatitis. Lancet 2017; 390:1007-1008. [PubMed: 28901924] This publication highlights the challenges in surgical trials for chronic pancreatitis.

15. Diener MK, Hüttner FJ, Kieser M, et al. Partial pancreatoduodenectomy versus duodenumpreserving pancreatic head resection in chronic pancreatitis: the multicentre, randomised, controlled, double-blind ChroPac trial. Lancet 2017; 390:1027-1037. [PubMed: 28901935] This multicenter clinical trial evaluates pancreaticoduodenectomy versus DPPHR.

16. Gurusamy KS, Lusuku C, Halkias C, Davidson BR. Duodenum-preserving pancreatic resection versus pancreaticoduodenectomy for chronic pancreatitis. Cochrane Database Syst Rev 2016; (2):CD011521. [PubMed: 26837472]

17. Jawad ZAR, Tsim N, Pai M, et al. Short and long-term postoperative outcomes of duodenum preserving pancreatic head resection for chronic pancreatitis affecting the head of pancreas: a systematic review and meta-analysis. HPB (Oxford) 2016; 18:121-128. [PubMed: 26902130]

18. Zhao X, Cui N, Wang X, Cui Y. Surgical strategies in the treatment of chronic pancreatitis: an updated systematic review and meta-analysis of randomized controlled trials. Medicine (Baltimore) 2017; 96:e6220; [PubMed: 28248878] This is a systematic review of randomized controlled trials evaluating the surgical management of chronic pancreatitis.

19. Bellin MD, Gelrud A, Arreaza-Rubin G, et al. Total pancreatectomy with islet autotransplantation: summary of an NIDDK workshop. Ann Surg 2015; 261:21-29. [PubMed: 25599324]

20. Harrison E, Lal S, McLaughlin JT. Enteroendocrine cells in gastrointestinal pathophysiology. Curr Opin Pharmacol 2013; 13:941-945. [PubMed: 24206752]

21. Sutherland DE, Radosevich DM, Bellin MD, et al. Total pancreatectomy and islet autotransplantation for chronic pancreatitis. J Am Coll Surg 2012; 214:409-424. [PubMed: 22397977]

22. Chinnakotla S, Bellin MD, Schwarzenberg SJ, et al. Total pancreatectomy and islet autotransplantation in children for chronic pancreatitis: indication, surgical techniques, postoperative management, and long-term outcomes. Ann Surg 2014; 260:56-64. [PubMed: 24509206]

23. Bellin MD, Beilman GJ, Dunn TB, et al. Islet autotransplantation to preserve beta cell mass in selected patients with chronic pancreatitis and diabetes mellitus undergoing total pancreatectomy. Pancreas 2013; 42:317-321. [PubMed: 23146918]

24. Bellin MD, Freeman ML, Schwarzenberg SJ, et al. Quality of life improves for pediatric patients after total pancreatectomy and islet autotransplant for chronic pancreatitis. Clin Gastroenterol Hepatol 2011; 9:793-799. [PubMed: 21683160]

25. Bellin MD, Kerdsirichairat T, Beilman GJ, et al. Total pancreatectomy with islet autotransplantation improves quality of life in patients with refractory recurrent acute pancreatitis. Clin Gastroenterol Hepatol 2016; 14:1317-1323. [PubMed: 26965843]

26. Bellin MD, Forlenza GP, Majumder K, et al. Total pancreatectomy with islet autotransplantation resolves pain in young children with severe chronic pancreatitis. J Pediatr Gastroenterol Nutr 2017; 64:440-445. [PubMed: 28231072] This publication reports on the outcomes after TPIAT for pediatric patients with severe refractory chronic pancreatitis.

-27. Colling KP, Bellin MD, Schwarzenberg SJ, et al. Total pancreatectomy with intraportal islet autotransplantation as a treatment of chronic pancreatitis in patients with CFTR mutations. Pancreas 2018; 47:238-244. [PubMed: 29206667] This study compares TPIAT patients with and without CFTR mutations. 
28. Bhutiani N, Cheadle GA, Bahr MH, Vitale GC. Comparative efficacy of bilateral thoracoscopic splanchnicectomy for intractable pain secondary to pancreatic cancer vs chronic pancreatitis. $\mathrm{J}$ Am Coll Surg 2017; 224(4):566-571. [PubMed: 28143718] This study reports on outcomes from 123 patients after splanchnicectomy from a single institution.

29. Bosanquet DC, Wilcox CR, Rasheed A. Bilateral thoracoscopic splanchnotomy to alleviate pain in chronic pancreatic disease. Ann Thorac Surg 2016; 101:e91-e93. [PubMed: 26897240]

30. Kuijpers M, Klinkenberg TJ, Bouma W, et al. Single-port videoscopic splanchnotomy for palliation of refractory chronic pancreatitis. Interact Cardiovasc Thorac Surg 2016; 22:393-396. [PubMed: 26794601] 


\section{KEY POINTS}

- $\quad$ Pursuing operative intervention early in the disease process results in improved outcomes.

- In recent studies, duodenum-preserving pancreatic head resection has been performed as well as pancreaticoduodenectomy in regards to patient outcomes.

- $\quad$ Each patient with chronic pancreatitis warrants careful consideration and an individualized approach in collaboration with multidisciplinary colleagues. 


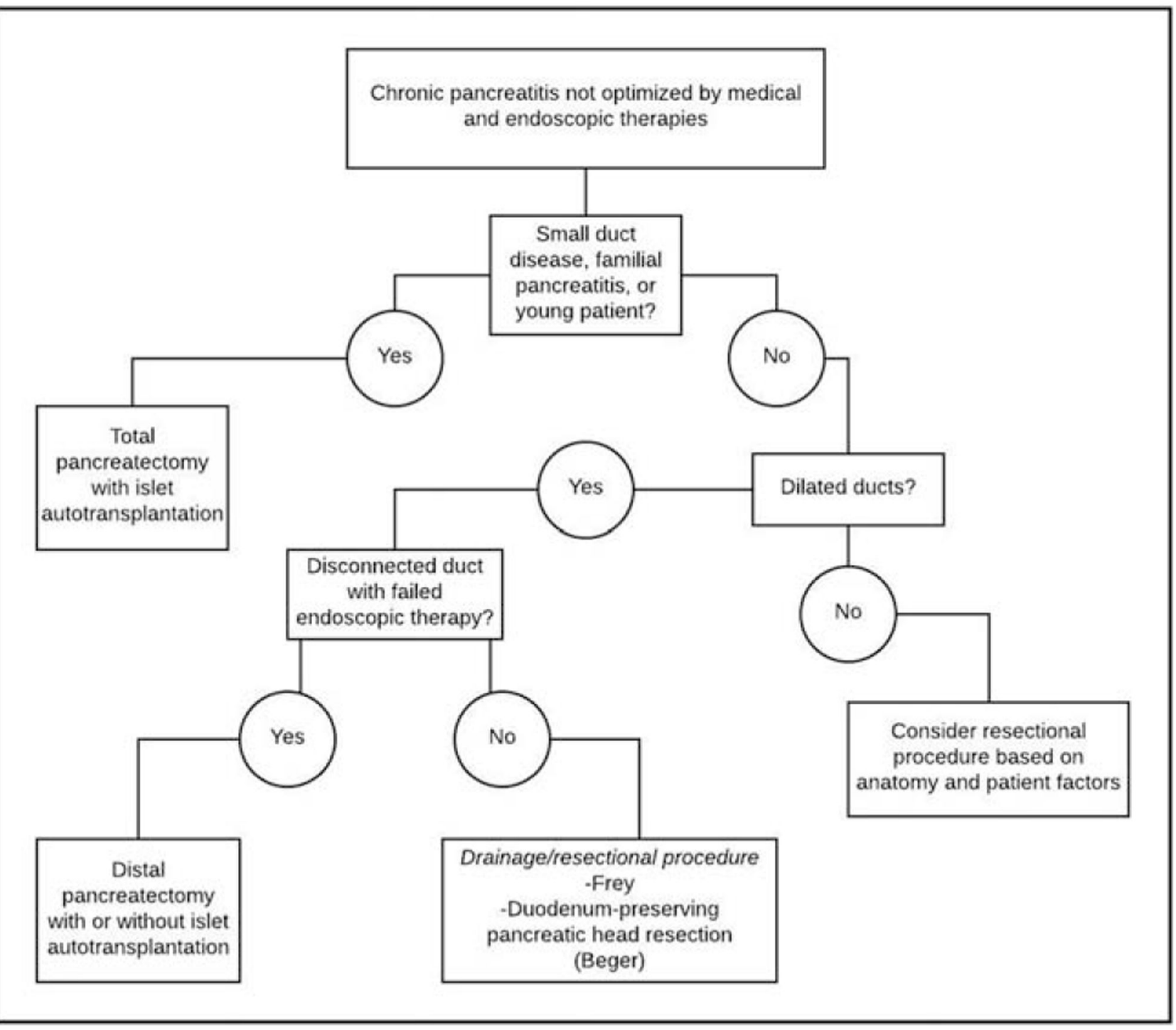

FIGURE 1.

Guide to surgical decision-making for the management of chronic pancreatitis. These are general guidelines only. Every patient warrants a tailored approach to care with multidisciplinary input. 


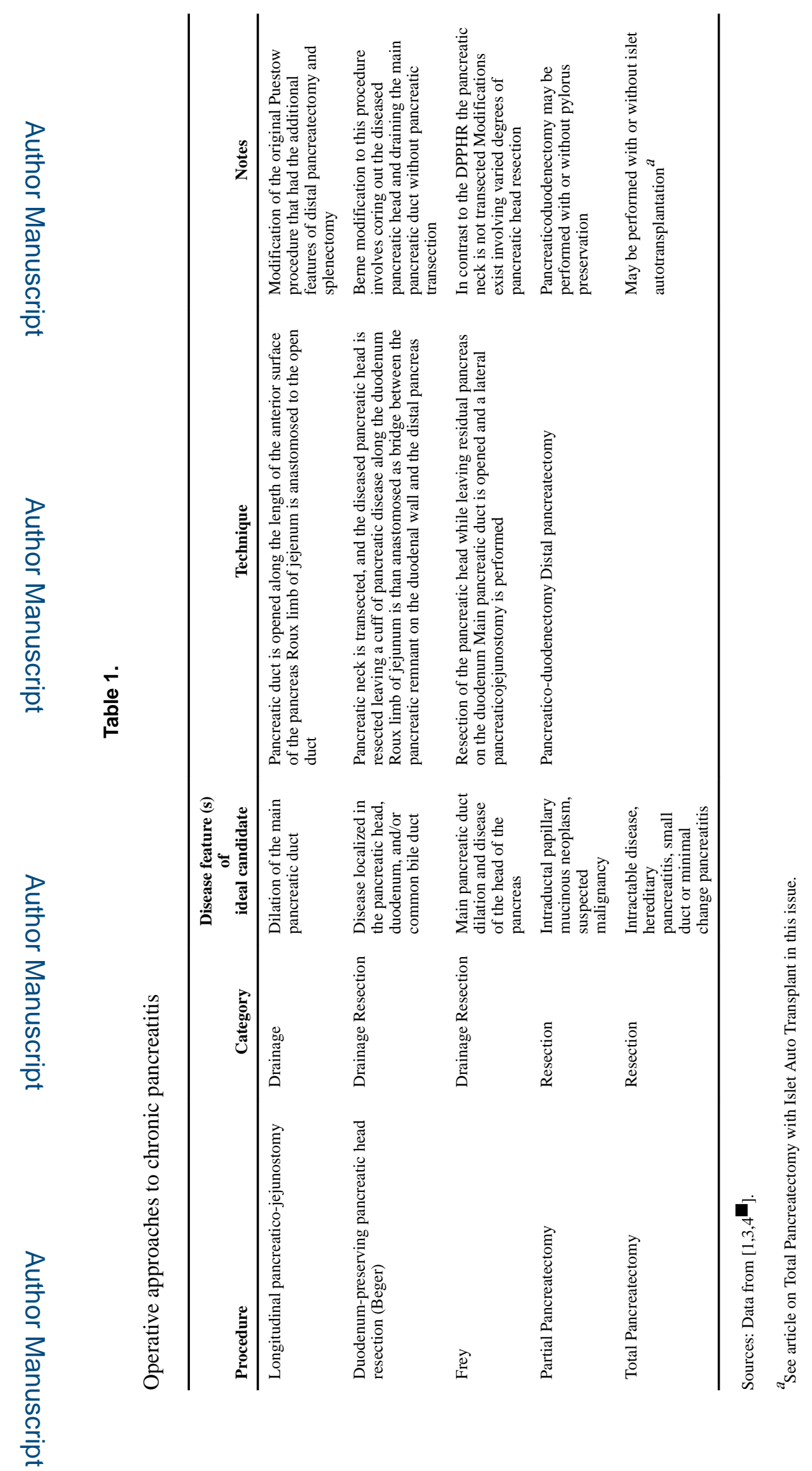

Curr Opin Gastroenterol. Author manuscript; available in PMC 2019 June 11. 